\title{
Amorphous Hydrocarbon Optical Properties
}

\author{
Anthony Jones \\ Université Paris Sud \& CNRS, Institut d'Astrophysique Spatiale, UMR8617, Orsay, F-91405 \\ email: Anthony.Jones@ias.u-psud.fr
}

\begin{abstract}
Hydrogenated amorphous carbon materials, a- $\mathrm{C}(: \mathrm{H})$, whose optical properties evolve in response to UV irradiation processing are promising candidate materials for cosmic carbonaceous dust. The optical properties of a: $\mathrm{C}(: \mathrm{H})$ particles have been derived as a function of size, band gap and hydrogen content over a wide wavelength range (EUV-cm) and can be used to investigate the size-dependent evolution of a-C(:H) material properties in the ISM.
\end{abstract}

Keywords. (ISM:) dust, extinction, ISM: molecules, ISM: general

\section{Modelling amorphous hydrocarbon optical properties}

The exact nature of interstellar carbonaceous dust is still something of a mystery. The evolution a-C:H, a-C or HAC solids is a complex subject that presents a particular challenge because these materials appear to be rather vulnerable to interstellar processing (e.g., Serra Díaz-Cano \& Jones 2008; Jones 2009; Jones \& Nuth 2011) and to undergo complex, size-dependent evolution arising, principally, from UV photon-driven processing. Here we introduce the $\operatorname{optEC}_{(\mathrm{s})}(\mathrm{a})$ model for the optical properties of amorphous hydrocarbons, a-C(:H), from hydrogen-poor, a-C, to hydrogen-rich, a-C:H, carbonaceous solids. These data provide a tool that can be used to explore carbonaceous dust and its observable characteristics. The optEC $(\mathrm{s})(\mathrm{a})$ model for a-C(:H) materials is presented in a series of papers (Jones 2012b,c,a), which derive their size-dependent structure and their complex refractive indices, $m(n, k))$. These data are publicly-available through the CDS (see the links in papers). We note that the derived data are strongly-constrained by the available laboratory data and have not been adjusted to fit astronomical observations. The upper panels in Fig. 1 show the parent or bulk material optEC $\mathrm{Cs}_{(\mathrm{s})}$ (a) values of $n$ and $k$, from EUV to mm wavelengths, as a function of the bulk material Tauc band gap, $E_{\mathrm{g}}$, and the lower panels in Fig. 1 show the equivalent $n$ and $k$ data for $1 \mathrm{~nm}$ radius particles. The major changes that occur as particle size decreases (see Jones 2012a) are: 1) increased surface hydrogenation and $\mathrm{CH}_{n}$ IR band intensities, and 2) a "collapse" of the continua for $\lambda \gtrsim 0.5 \mu \mathrm{m}$ and $E_{\mathrm{g}} \lesssim 1.5 \mathrm{eV}$. As shown by Jones $(2012 \mathrm{a})$, the latter effect is due to a reduction in the maximum-allowable, particle-radius-determined aromatic domain sizes as the particle radius decreases, which is clearly seen in the lower panels in Fig. 1. For particles with radii $<1 \mathrm{~nm}$ the optical properties begin to look rather similar.

As has been shown the derived $\operatorname{optEC}_{(\mathrm{s})}(\mathrm{a})$ data are qualitatively consistent with: the FUV-UV bump-visible extinction (non-)correlations, the IR absorption and emission bands in the $3.3-3.6 \mu \mathrm{m}$ region, variations in the FIR-mm emissivity index (Jones $2012 \mathrm{~b}, \mathrm{c}, \mathrm{a})$. Further, the model predicts that: the $3.28 \mu \mathrm{m}$ aromatic $\mathrm{CH}$ band will always be accompanied by aliphatic $\mathrm{CH}_{n}$ bands and/or a plateau in the $3.35-3.55 \mu \mathrm{m}$ region, the end of the road evolution for small a- $\mathrm{C}(: \mathrm{H})$ particles is probably aromatic/aliphatic cage-like structuresthat could provide a route to fullerene formation (Bernard-Salas et al. 2012; Micelotta et al. 2012) the UV-photolytic fragmentation of small a-C:H grains will lead to the formation of small hydrocarbon molecules ( $\mathrm{CCH}, \mathrm{c}_{-} \mathrm{C}_{3} \mathrm{H}_{2}, \mathrm{C}_{4} \mathrm{H}$, etc.) in PDR 

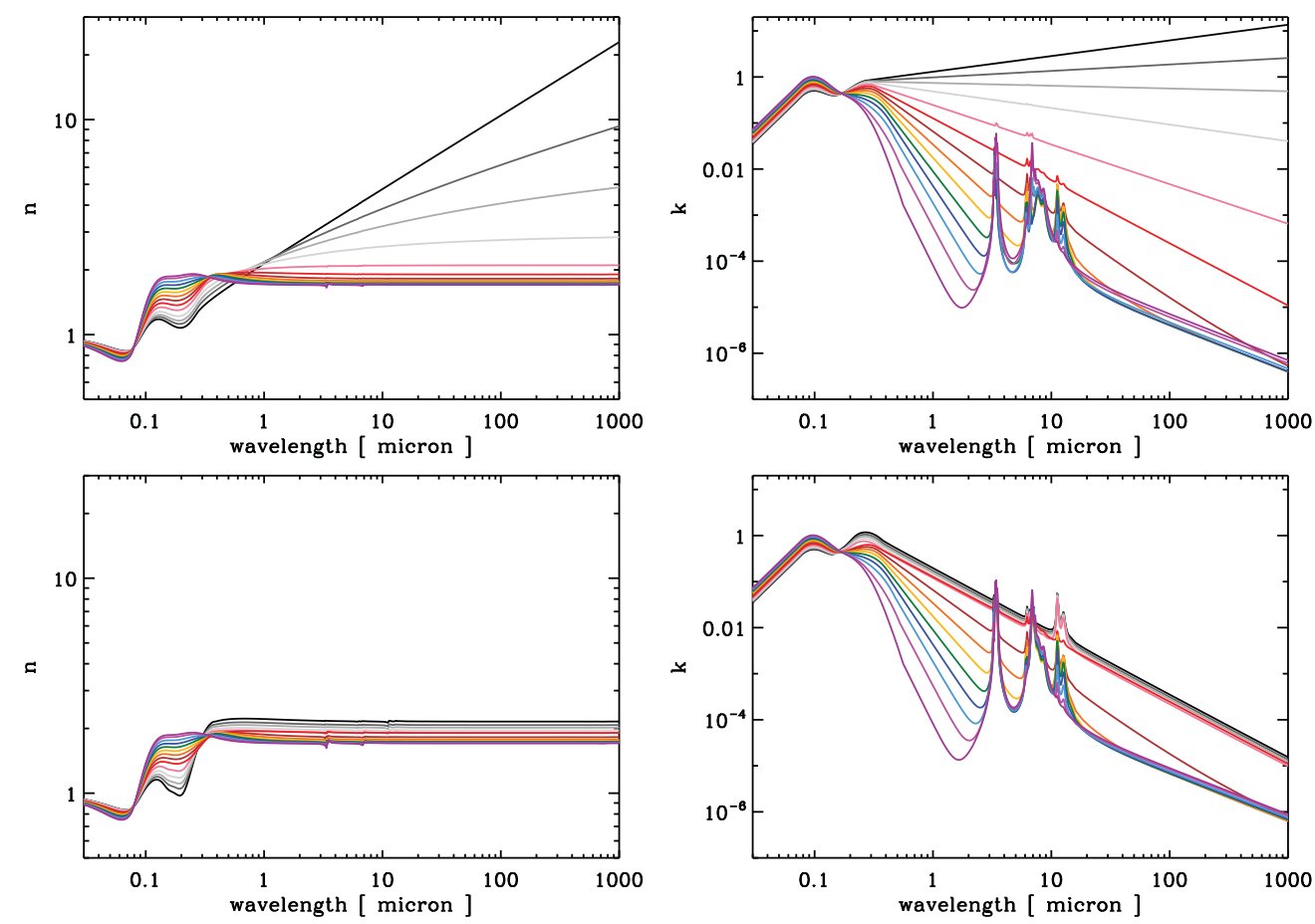

Figure 1. The optEC $(\mathrm{s})$ (a) model real and imaginary parts of the complex refractive index, $n$ and $k$, for bulk a-C:H materials ( $\equiv a>100 \mathrm{~nm}$, upper) and for $a=1 \mathrm{~nm}$ particles (lower). The bulk material band gap, $\mathrm{E}_{\mathrm{g}}$, increases from $-0.1 \mathrm{eV}$ (top) to $2.67 \mathrm{eV}$ (bottom) at $\lambda \sim 2 \mu \mathrm{m}$.

regions, and "pure" graphite grains and "perfect" PAHs are probably not important components of dust in the ISM (Jones 2012b,c,a).

\section{Concluding remarks}

A new data-set for the size-dependent optical properties of amorphous hydrocarbon particles, for the first time, provides a means to a detailed exploration of the evolution of these complex materials in the ISM. The principal drivers of their evolution would appear to be photon-induced processing, which results in de-hydrogenation and band gap closure that are coupled to significant changes in their IR spectra and their long wavelength emission.

\section{References}

Bernard-Salas, J., Cami, J., Peeters, E., et al. 2012, ApJ 757, 41

Jones, A. P. 2009, in Astronomical Society of the Pacific Conference Series, Vol. 414, Cosmic Dust - Near and Far, ed. T. Henning, E. Grün, \& J. Steinacker, 473-+

Jones, A. P. 2012a, A\& A 542, A98

Jones, A. P. 2012b, A\&A 540, A1

Jones, A. P. 2012c, $A \mathscr{E} A$ A 540, A2

Jones, A. P. \& Nuth, J. A. 2011, A\&A 530, A44

Micelotta, E. R., Jones, A. P., Cami, J., et al. 2012, ApJ 761, 35

Serra Díaz-Cano, L. \& Jones, A. P. 2008, A $\& A$ 492, 127 